\title{
Embolisation of a traumatic aneurysm of the posterior circumflex humeral artery in a volleyball player
}

\author{
M Vlychou, G Spanomichos, A Chatziioannou, M Georganas, G M Zavras
}

\begin{abstract}
Repetitive minor vascular injuries caused by physical activity in athletes may lead to ischaemia of the upper extremities. In volleyball players in particular, traumatic aneurysm of the posterior circumflex humeral artery has been reported to be a cause of ischaemia of the arm and hand. Such an aneurysm is described here; it was treated successfully with endovascular embolisation.
\end{abstract}

(Br F Sports Med 2001;35:136-137)

Keywords: aneurysm; embolisation; posterior circumflex humeral artery; ischaemia; upper extremities; volleyball

The presence of an aneurysm of the posterior circumflex humeral artery (PCHA) is a rare entity found in volleyball players. ${ }^{12}$ It is believed that the throwing motions of the dominant shoulder in volleyball players may cause minor repetitive traumas in the arterial wall of the axillary artery or its tributaries, which can lead to a traumatic aneurysm. Although the usual method of treatment of such aneurysms is surgical resection, embolisation has been performed as an alternative. ${ }^{12}$

\section{Case report}

A 19 year old, right handed, male volleyball player was referred to the department of vascular surgery. He had been an amateur player since the age of 14 and a professional for the preceding year. He presented with recurrent episodes of a painful right hand over the preceding 10 days. The onset of pain was sudden and occurred during a stressful volleyball match. Clinical examination disclosed coolness and paleness of the right limb, particularly at the distal end, with dysaesthesia in the fingers and absence of pulsation of the right radial and ulnar artery. The patient had no past clinical history and had never taken any type of medication.

He underwent laboratory examination and electrocardiographic monitoring; the results were normal. The chest $x$ ray film showed no evidence of cervical ribs. Examination with the Doppler probe showed diminished blood flow in the distal part of the radial and ulnar artery. Angiography of the aortic arch and both arms was performed by the Seldinger technique. The aortic arch, the proximal brachiocephalic vessels, and the arteries of the left arm and hand showed no abnormalities. Angiography of the right upper limb was abnormal, showing peripheral emboli at the origin of the common interosseous artery and the distal end of the radial artery. As a result, the superficial palmar arch of the affected hand was shown to supply the deep palmar arch by reversed flow. The source of the clots was an irregular aneurysm at the origin of the right PCHA (fig 1A) which was clearly identified by angiography. We decided to perform immediate therapeutic embolisation. The embolic agents used were five spiral Gianturco coils (four coils $5 \mathrm{~cm} \times 8$
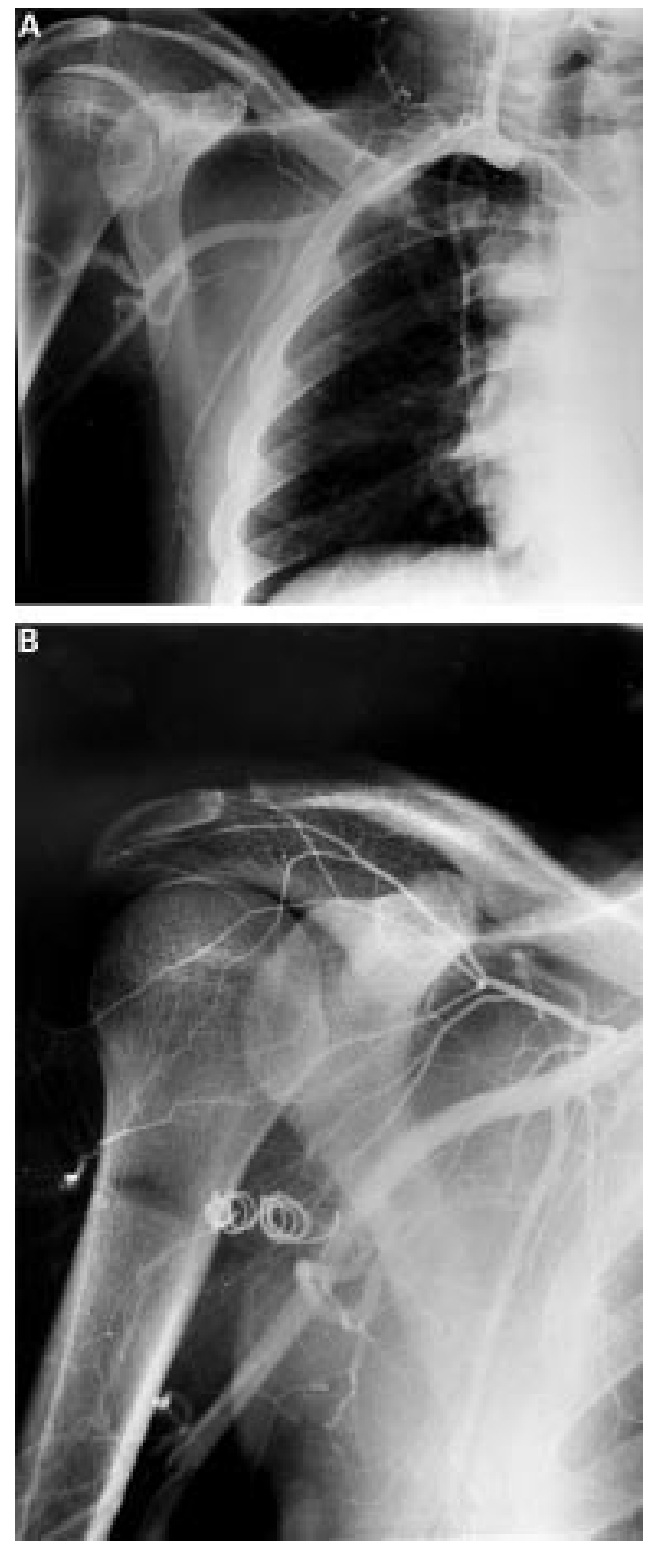

Figure 1 Radiographs during angiography showing the aneurysm of the posterior circumflex humeral artery $(A)$ and total occlusion of the aneurysm after embolisation with Gianturco coils (B). 
$\mathrm{mm}$ and one coil $5 \mathrm{~cm} \times 5 \mathrm{~mm}$; Cook, Denmark), which created total occlusion of the PCHA (fig 1B). Placement of coils on both sides of the aneurysm in a distal to proximal arrangement ensured appropriate filling of its sac and therefore occlusion of the aneurysm from the circulation.

On the next day, the patient underwent embolectomy at the site of the peripheral emboli in the common interosseous and radial artery, with extraction with a Fogarty catheter. This operative treatment allowed normal arterial flow with complete remission of the ischaemic symptoms of the right hand. A second selective angiography was performed as a follow up three months later which showed continued total occlusion of the right PCHA, perfect exclusion of the aneurysm, and patency of all the other proximal and distal arteries of the right upper extremity.

\section{Discussion}

The posterior circumflex humeral artery is considerably larger than the anterior and arises from the third part of the axillary artery. It winds around the surgical neck of the humerus, through the quadrangular space, and distributes branches to the shoulder joint, deltoid, teres major and minor, and the long and lateral heads of the triceps. ${ }^{3}$

In our patient, the aneurysm of the PCHA was considered to be the cause of the peripheral ischaemia. It is assumed that the mechanism responsible is repetitive minor trauma to the vessel wall caused by the throwing motion of the shoulder. ${ }^{4-6}$ The humeral head articulates with the glenoid cavity of the scapula in a ball and socket joint and only a portion of it is in contact with the cavity in any one position of the arm. Skeletally the shoulder joint is weak, because, for such strength as it possesses, it is dependent on the support given by the muscles that surround it. Nevertheless, the glenoidal labrum, the fibrous capsule, and the other ligaments of the shoulder joint allow a considerable amount of movement in three planes and appreciable rotation of the humerus around its long axis. Muscular pull on the humerus by the pectoralis major muscle may cause downward subluxation of the humeral head, as the result of violent abduction and upward rotation of the shoulder, so that the strong tendon of the pectoralis major muscle is stretched. ${ }^{1}$ The arm of a volleyball player is swung upwards and then downwards with maximum force innumerable times a day, and, in particular, external rotation and hyperabduction of the arm seem to have a stretching effect on the PCHA as it winds around the surgical neck of the humerus. ${ }^{6}$ This leads to localised microtrauma of the wall of the PCHA at the site of contact with the humeral head and subsequent aneurysm formation due to chronic irritation of the arterial wall. ${ }^{6}$ Entrapment of the PCHA in the quadrilateral space does not seem to be a likely cause of this aneurysm, because it developed at the origin of the PCHA, while the midsection of the artery is traversing the quadrilateral space. In addition, the main symptoms of the patient were not those of axillary nerve entrapment. ${ }^{1}$ The aneurysm remains occult as long as the player is free of symptoms. The embolic complications of the affected extremity, in combination with pain and ischaemia, led to the manifestation of the entity. ${ }^{12}$

The diagnosis may be suggested by magnetic resonance angiography, sonography being impractical in this area because of the posterior location of the vessel. However, diagnosis should be confirmed by selective angiography. Furthermore, interventional procedures may be used for the treatment of the clot such as thrombolysis, if the thrombus is fresh, or embolisation of the aneurysmal sac, to prevent further distal emboli. We decided to embolise the aneurysm, because selection of the artery was easy and deployment of the coils was safe, producing permanent exclusion of the sac. ${ }^{12}$ Spontaneous thrombosis of the aneurysm as a future alternative was ruled out, because the player was very active and in the middle of his career, and the sequelae of future emboli may be devastating. ${ }^{78}$

Given the serious consequences of this pathology in this particular subgroup of athletes, every suspicious symptom warrants investigation to exclude the presence of such an aneurysm.

1 Reekers JA, den Hartog BM, Kuyper CF, et al. Traumatic aneurysm of the posterior circumflex humeral artery: a vol-

2 Reekers JA, Koedam N. Volleyball-related ischemia of the hand. Cardiovasc Intervent Radiol 1998;21:261.

3 Gray's anatomy. 36th ed. Edinburgh: Churchill and Livingstone, 1980:700-2.

4 Baker LC, Liu SH. Neurovascular injuries to the shoulder. Review. F Orthop Sports Phys Ther 1993;18:360-4.

5 McCarthy WJ, Yao JS, Schafer MF, et al. Upper extremity arterial injury in athletes. $\mathcal{F}$ Vasc Surg 1989;9:317-27.

6 Durham JR, Yao JS, Pearce WH, et al. Arterial injuries in the thoracic outlet syndrome. $\mathcal{F}$ Vasc Surg 1995;21:57-70.

7 Kee ST, Dake MD, Wolfe-Johnson B, et al. Ischemia of the throwing hand in major league pitchers: embolic occlusion from aneurysms of the axillary artery branches. $f$ Vasc Interv Radiol 1995;6:979-82.

8 Nijhuis HH, Moeller-Wiefel H. Occlusion of the brachial artery by thrombus dislodged from a traumatic aneurysm of the anterior humeral circumflex artery. $\mathcal{F}$ Vasc Surg 1991; 13:408-11.

Take home message

Traumatic aneurysm of the PCHA is an uncommon but serious cause of ischaemia of the upper extremity. Diagnostic angiography in combination with embolisation of the aneurysm is an effective alternative method of management to surgical resection. 